\title{
Morphological study of the vastus medialis oblique in recurrent patellar dislocation based on magnetic resonance images
}

Lei Shu, Xu Yang, Hangyuan He, Biao Chen, Liaobin Chen ${ }^{*}$ and Qubo Ni*

\begin{abstract}
Background: To investigate the morphological parameters of the vastus medialis obliquus (VMO) muscle and delineate its importance in the maintenance of patellofemoral joint stability.

Methods: The magnetic resonance imaging data of seventy-five knees (fifty-four patients) with recurrent lateral patella dislocation (LPD) and seventy-five knees (seventy patients) without recurrent LPD were retrospectively analysed. Five morphological parameters related to the VMO (elevation in the sagittal plane and coronal plane, craniocaudal extent, muscle-fibre angulation, cross-sectional area ratio) and two patella tilt parameters (patella tilt angle, bisect offset ratio) were measured in MR images. The independent-samples t test or chi-square test was used for statistical comparisons.

Results: The mean ages of the patients in the recurrent LPD group and control group were $22.1 \pm 9.9$ years and $24.0 \pm 6.5$ years, respectively. Eighteen out of seventy-five (24\%) patients MRI showed VMO injuries. Compared with the control group, the patients with recurrent LPD showed significantly higher sagittal VMO elevation $(10.4 \pm 2.3 \mathrm{~mm}$ vs. $4.1 \pm 1.9 \mathrm{~mm}$ ), coronal VMO elevation $(15.9 \pm 5.7 \mathrm{~mm}$ vs. $3.9 \pm 3.7 \mathrm{~mm})$, muscle-fibre angulation $\left(35.4 \pm 8.0^{\circ} \mathrm{vs}\right.$. $\left.27.9 \pm 6.3^{\circ}\right)$, patella tilt angle $\left(25.9 \pm 10.7^{\circ}\right.$ vs. $\left.9.1 \pm 5.2^{\circ}\right)$, and bisect offset ratio values $(0.9 \pm 0.3$ vs. $0.5 \pm 0.1)$ and significantly lower craniocaudal extent $(13.7 \pm 5.3 \mathrm{~mm}$ vs. $16.7 \pm 5.1 \mathrm{~mm})$ and cross-sectional area ratio values $(0.05 \pm 0.02$ vs. $0.07 \pm 0.02)$.
\end{abstract}

Conclusions: The results showed that abnormalities in the VMO and patella tilt were clearly present in recurrent LPD patients compared with normal people.

Keywords: Recurrent lateral patellar dislocation, Vastus medialis obliquus, Magnetic resonance imaging, Morphological parameters

\section{Background}

Recurrent lateral patellar dislocation (LPD) is usually secondary to primary acute patellar dislocation and mostly occurs in young people aged 10-17 years [1]. The incidence of primary acute patella dislocation in the general population is $7-49$ cases per $100,000[2,3]$. With nonoperative management, the rate of recurrent LPD after

*Correspondence: Ibchen@whu.edu.cn; niqubo@znhospital.cn Department of Orthopedic Surgery, Zhongnan Hospital of Wuhan University, Wuhan 430071, China acute patellar dislocation has been reported to be as high as $44 \%$ [4]. Recurrent LPD often causes symptoms including persistent pain, knee weakness and mechanical limitations [5].

Patellofemoral joint stability is maintained by both bone and soft tissue stabilizers. Numerous studies have investigated the effect of osseous factors on LPD, but the influence of soft tissue factors is still being explored. Soft tissues can be considered either active structure stabilizers (quadriceps femoris) or passive structure stabilizers (ligaments), which stabilize the patellofemoral joint 
together during knee flexion [6]. The medial patellofemoral ligament (MPFL) accounts for $50-60 \%$ of the total limiting force against LPD, which is generally considered to be the most important soft tissue in the medial region of the patellofemoral joint $[1,7,8]$. However, several scholars have confirmed that the quadriceps muscle also plays an imperative role in maintaining the stability of the patella [9-12]. The vastus medialis obliquus (VMO) muscle seems to be an important dynamic stabilizer for neutralizing the lateral force of the patella, and its importance is gradually being recognized $[9,13,14]$. In general, the lateral pull of the larger vastus lateralis (VL) is counterbalanced by the force of the VMO to ensure patellar stability. When there is an imbalance, abnormal lateral tracking of the patella may occur. The disruption of this mechanical balance between the VMO and VL has frequently been attributed to an insufficiency of the VMO secondary to atrophy and hypoplasia [15]. Due to the anatomical relationship and characteristics of the MPFL and VMO, MPFL tears are usually accompanied by VMO injuries, while most MPFL tears occur on the femur side. This may cause the femoral attachment point of the VMO to elevate in the sagittal and coronal planes, decreasing the dynamic medial stabilizing force. Unfortunately, VMO injuries occurs in approximately 45-93\% of primary patella dislocation patients [16], which may lead to secondary atrophy of the VMO.

To our knowledge, no studies have comprehensively described the morphological characteristics of the VMO in patients with recurrent LPD. Magnetic resonance imaging (MRI) is the gold standard for assessing soft tissue and can clearly show the contours of muscles [17]. Therefore, the purpose of our study was to investigate the difference in VMO-related morphological parameters assessed by MRI between patients with recurrent LPD and a control group.

\section{Materials and methods}

All patients undergoing MRI examinations in our hospital from June 2018 to June 2020 were selected. First, the two keywords of "patella dislocation" and "no obvious abnormality of knee joint" were used to search MR reports in the picture archiving and communications system (PACS) workstation (Centricity, GE Healthcare, St. Gilles, United Kingdom) to initially screen patients. Then, on the basis of both their medical histories and previous medical records. Two head doctors with more than three years of work experience in joint and sports medicine screened the subjects according to the following inclusion and exclusion criteria. A total of fifty-four patients with recurrent LPD (seventy-five knees) and seventy controls (seventy-five knees) were enrolled. Moreover, age and sex were matched as closely as possible between the two groups.

The inclusion and exclusion criteria for the patients with recurrent LPD were as follows:

Inclusion criteria: (1) Recurrent LPD was diagnosed by two senior doctors in the joint and sports medicine department according to the patient's history, physical examination findings and MRI findings. (2) The patient was not previously treated in the rehabilitation department or receive any special training related to strengthening the quadriceps muscles. (3) MRI images were taken within 10 days after the recurrence of LPD.

Exclusion criteria: (1) Patients with primary patellar dislocation. (2) Traumatic patellar dislocation occurred as a result of direct trauma to the medial patella or a fall onto the knee joint with concomitant patellar dislocation. (3) Patients with any preexisting knee disorders, previously underwent knee surgery, had a fracture of the distal femur or tibial head, or had a multi-ligament injury. (4) Patients with history of a neuromuscular disease (e.g., polio). (5) Patients with obvious effusion of the knee joint.

The inclusion and exclusion criteria for the control group were as follows: MRI examination of the knee was performed for people to exclude diseases because of knee discomfort and no significant structural damage (e.g., fractures) or anatomical abnormalities (e.g., osteoarthritis) was reported.

Sagittal, coronal, and transverse MR images were obtained in all patients. Two doctors in joint and sports medicine measured the following five parameters related to the VMO (elevation on sagittal plane and coronal plane, craniocaudal extent, muscle-fibre angulation, cross-sectional area ratio) and two patella tilt parameters (patella tilt angle, bisect offset ratio) in both groups. The type of femoral trochlear dysplasia present in each patient was recorded according to the classification system reported by Dejour et al. [18] and Lippacher et al. [19] on axial MR images: type A, shallow trochlea and a subchondral sulcus angle $>145$ degrees; type B, flat or convex trochlea; type $\mathrm{C}$, asymmetry of trochlear facets with a hypoplastic medial facet; type $\mathrm{D}$, asymmetry of trochlear facets or cliff pattern, it was further categorized as normal or low-grade (type A), or high-grade dysplasia (type $\mathrm{B}, \mathrm{C}$, or $\mathrm{D}$ ). Moreover, the diagnosis of the VMO injury was recorded according to the criteria reported by Elias et al. [20]. Except for the cross-sectional area, which was calculated by ImageJ freeware, the parameters were measured by the PACS workstation. All the parameters were repeatedly measured within an interval of two weeks. The MRI (Philips MR Systems Ingenia 3.0T, Andover, Massachusetts) protocols used in our hospital were described in our previous study [21]. All patients were in a supine position, with a standard knee 
coil center level against the lower edge of the patella. The knee and hip joint naturally extended, and the feet were braced to prevent any movement. Our MRI protocol includes: (1) coronal proton density weighted spectral attenuated inversion recovery (PDW-SPAIR) MR images [repetition time msec (TR)/echo time msec (TE) 1940/30, field of view (FOV) $220 \mathrm{~mm} \times 179 \mathrm{~mm}$, matrix $368 \times 245$, slice thickness $3 \mathrm{~mm}$, sections per slab 21]; (2) transverse PDW-SPAIR MR images (TR/TE 2036/30, FOV $169 \mathrm{~mm} \times 189 \mathrm{~mm}$, matrix $344 \times 264$, slice thickness $4 \mathrm{~mm}$, act slice gap $0.4 \mathrm{~mm}$, sections per slab 24); (3) sagittal T1-weighted aTSE (turbo spin-echo) MR images (TR/TE 694/12, FOV $160 \mathrm{~mm} \times 160 \mathrm{~mm}$, matrix $308 \times 240$, slice thickness $3 \mathrm{~mm}$, act slice gap $0.3 \mathrm{~mm}$, sections per slab 24); (4) sagittal proton density weighted spectral inversion recovery (PDW-SPIR) MR images (TR/ TE $1,554 / 30$, FOV $160 \times 160 \mathrm{~mm}$, matrix $292 \times 231$, slice thickness $3 \mathrm{~mm}$, act slice gap $0.3 \mathrm{~mm}$, sections per slab 24).

\section{MR measurements}

\section{The measurement of $\mathrm{VMO}$ elevation}

The VMO elevation was measured in the sagittal and coronal planes according to Zhang et al's [22] measurement method. In brief, the transverse slice in which the adductor tubercle could clearly be seen was defined as the optimally measurable slice, as indicated by a blue line. In this transverse image, the corresponding sagittal and coronal planes were identified (Fig. 1).
On the selected sagittal slice, the apex of the anterosuperior border of the bone cortex of the adductor tubercle was set as the starting point. VMO elevation was defined as the shortest distance from the starting point extending obliquely to the inferior edge of the muscle belly. On the selected coronal slice, the apex of the medial superior border of the adductor tubercle was set as the starting point. VMO elevation in the coronal plane was defined as the vertical distance from the starting point to the inferior margin of the $\mathrm{VMO}$ muscle (Fig. 1).

\section{The measurement of muscle-fibre angulation and craniocaudal extent of the VMO}

First, the "Roman arch" was most obvious in the axial plane, and the corresponding sagittal slice was selected (Fig. 2a, d). Two concentric circles were drawn on the proximal and distal sides of the femur, and the line passing through two centers was taken as the longitudinal axis of the femoral shaft. Second, the adductor tubercle was found on the transverse slice, and the corresponding sagittal slice was located to determine the lowest point of the VMO. The VMO muscle-fibre angulation, the angle between the VMO muscle-fibre and the longitudinal axis of femoral shaft, was measured in the sagittal plane (Fig. 2b, e). The lowest point of VMO was located in this plane, and the corresponding horizontal line was established in the sagittal plane central to the patella longitudinal axis. The craniocaudal extent of the VMO was defined as the vertical distance from this horizontal line to the proximal patellar pole (Fig. 2c, f).

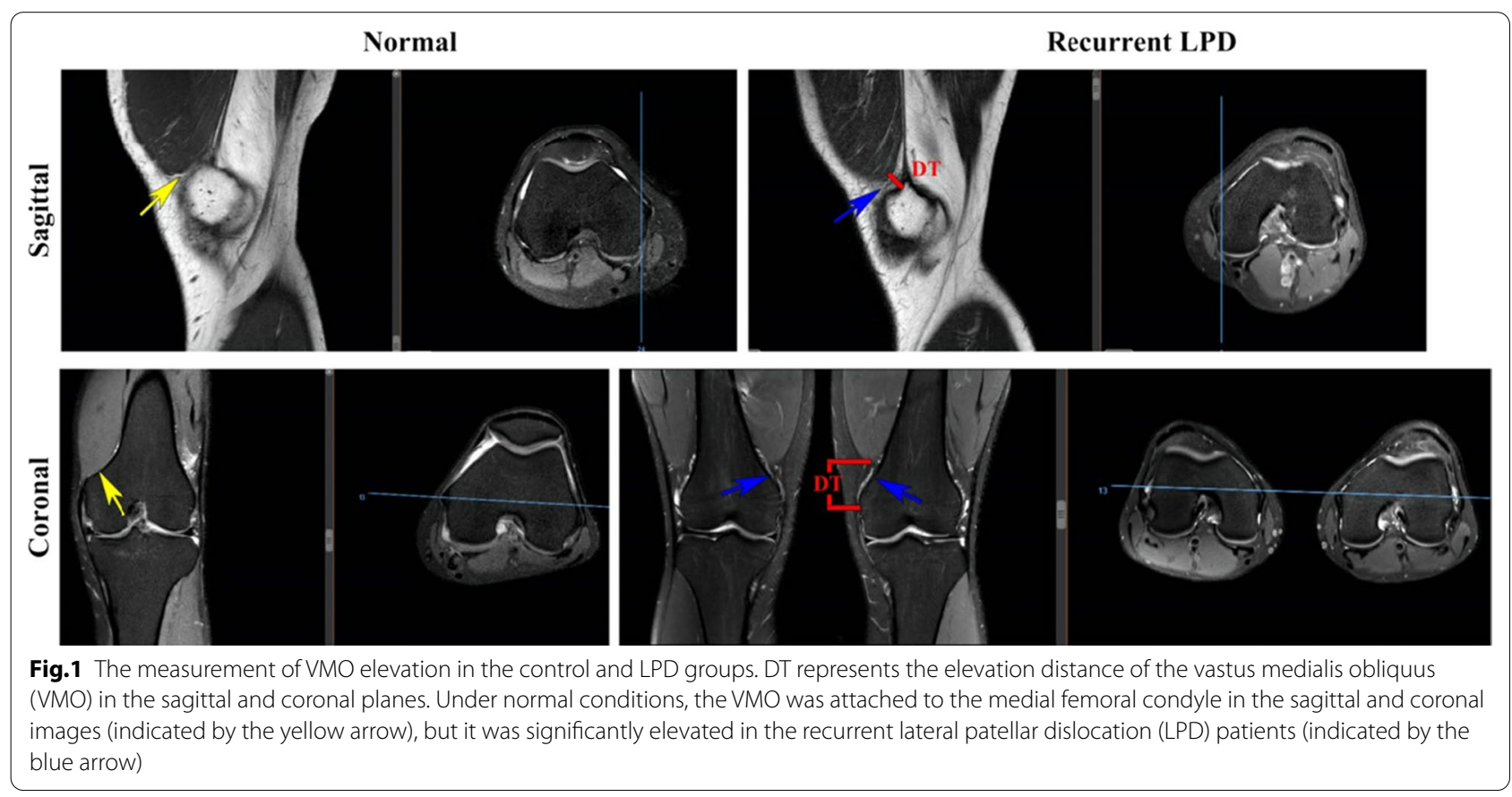



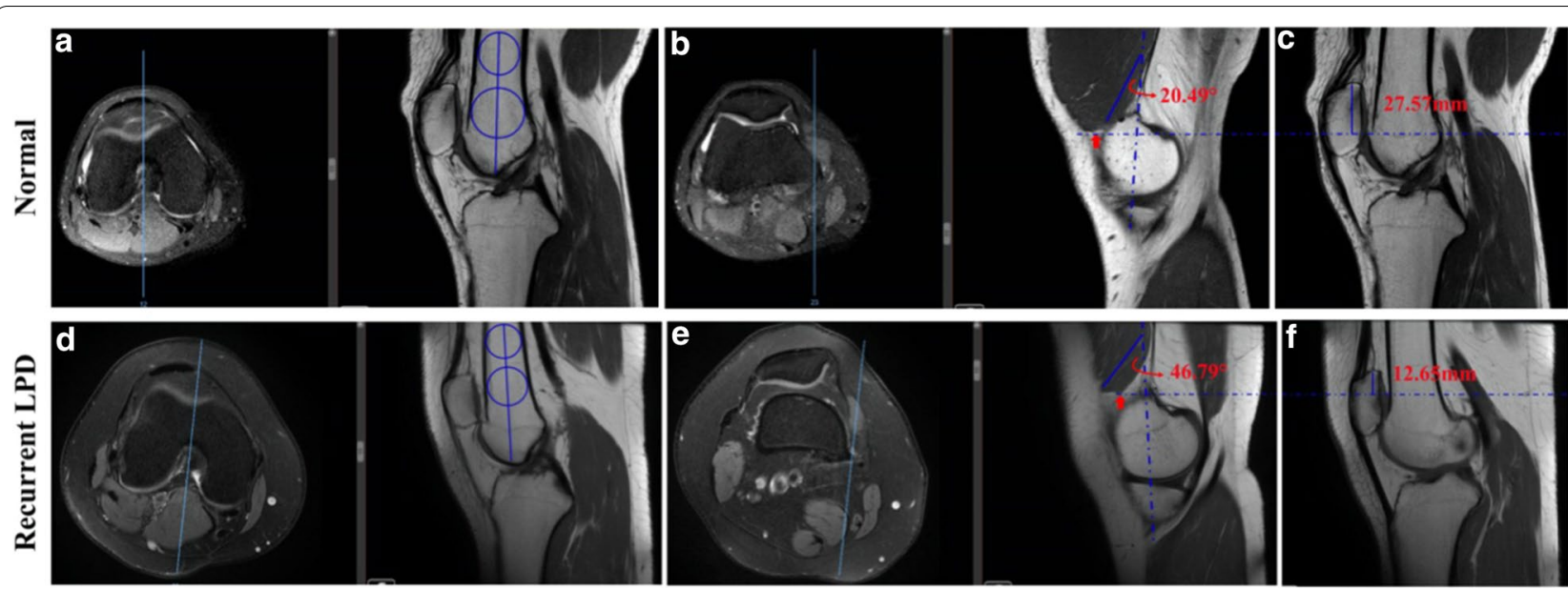

Fig. 2 The measurement of muscle fibre angulation and craniocaudal extent of the VMO in the control and recurrent LPD groups. a, $\mathbf{d}$ Images showing the plane in which the "Roman arch" was most obvious; the longitudinal axis of the femoral shaft was identified; $\mathbf{b}$, e The adductor tubercle was located in the medial margin of the femur in the transverse slice; the red dots indicate the lowest point of the VMO, and the VMO muscle-fibre angulation in the controls and LPD patients were $20.49^{\circ}$ and $46.79^{\circ} ; \mathbf{e}$, f The magnitudes of craniocaudal extent of the VMO in the controls and LPD patients were $27.57 \mathrm{~mm}$ and $12.65 \mathrm{~mm}$, respectively

\section{The measurement of the cross-sectional area ratio of the VMO}

According to the method introduced by Balcarek et al. [23]. First, the longitudinal axis of the patella was established in the central sagittal plane. In this sagittal image, the corresponding transverse slice located at the proximal patellar pole and the adjacent slice located above and below this reference slice were identified. Then the crosssectional area of the VMO and the whole thigh were calculated on these three slices respectively (Fig. 3). The cross-sectional area ratio of the VMO was defined as the ratio between the cross-sectional area of the VMO and the whole thigh. Finally, the mean cross-sectional area ratio among the three slices was obtained.

\section{The measurement of the patella tilt angle and patella offset index}

The transverse plane, which allows the visualization of the intact "Roman arch" and posterior femoral condyles, was selected. The posterior condylar reference line was drawn tangent to the posterior femoral condyles. The patella tilt angle was formed by the line along the width of the maximal patella and the line along the posterior femoral condyle (Fig. 4).

According to the method described by Christopher et al. [24] and Callaghan et al. [25], a line was drawn through the deepest portion of the trochlear groove and perpendicular to the posterior condylar reference line. The intersection of this line and the line along the width of the maximal patella was defined as point $O$. In the transverse plane of the widest layer of the patella, the innermost point of the patella was defined as point $\mathrm{A}$, and the outermost point was defined as point B (Fig. 4). The ratio of $\mathrm{OB} / \mathrm{AB}$ was defined as the bisect offset ratio.

\section{Statistical analysis}

SPSS 22.0 (IBM Corp. Released 2013. IBM SPSS Statistics for Windows. Armonk, NY: IBM Corp) was used to assess the relevant data. All parameters are presented as the mean \pm standard deviation. The continuous and categorical variables were compared between the two groups were analyzed by the independent-samples $t$ test and chi-square test, respectively. $P<0.05$ was considered statistically significant. Moreover, the intraclass correlation coefficient (ICC) was also analysed for duplicated measurements taken by two observers.

\section{Results}

Fifty-four patients (seventy-five knees) diagnosed with recurrent LPD were enrolled in our study. The study group consisted of eighteen males and thirtysix females. The average age of the patients was $22.11 \pm 9.87$ years (range, $12-45$ years), and their average BMI was $24.1 \pm 3.6 \mathrm{~kg} / \mathrm{m}^{2}$. In addition, seventy controls (seventy-five knees) were recruited. The baseline characteristics of the two groups are presented in Table 1. Compared with the control group, the recurrent LPD group showed more severe femoral trochlear dysplasia, as indicated by a higher grade (Table 1). Eighteen out of seventy-five (24\%) patients MRI showed VMO injuries, and VMO tear was present in 


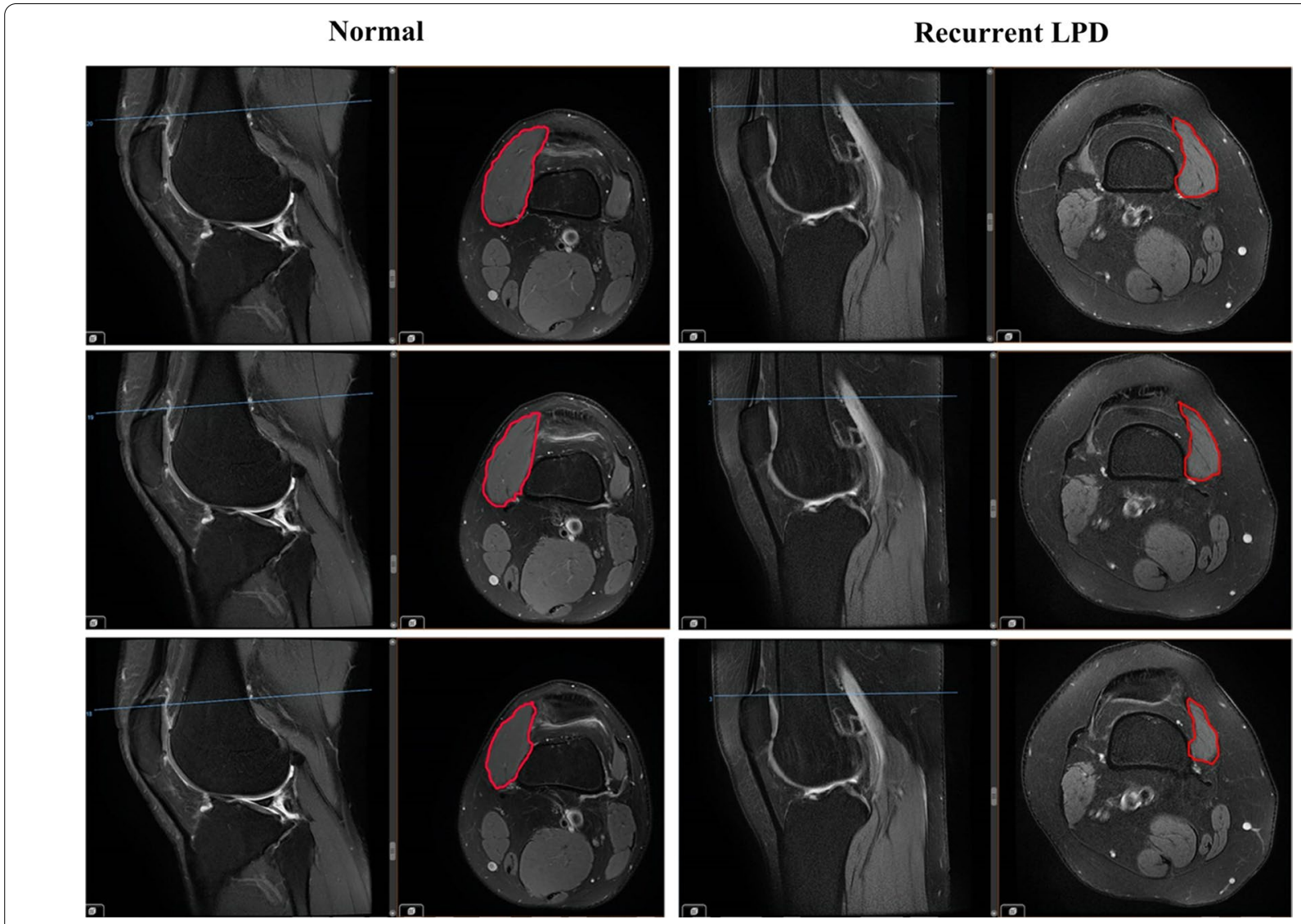

Fig. 3 The measurement of the cross-sectional area ratio of the VMO in the control and recurrent LPD groups. The three solid blue lines correspond to the three adjacent transverse slices, and the part indicated by the red line is the cross-sectional area of the VMO

only one patients, oedema signal was observed in thirteen patients and haemorrhage signal was found in the other four patients. Additionally, the intraclass correlation coefficients indicated excellent inter- and intraobserver agreement for all the variables $(>0.75)$.

As shown in Fig. 1, the VMO was attached to the medial femoral condyle in the sagittal and coronal sectional images in the control group, but it was significantly elevated in the LPD patients. In addition, the patellofemoral joint was in good alignment in the controls. The mean magnitudes of sagittal and mean coronal VMO elevations were significantly higher in the recurrent LPD group than in the control group $(10.4 \pm 2.3 \mathrm{~mm}$ vs. $4.1 \pm 1.9 \mathrm{~mm}$, $15.9 \pm 5.7 \mathrm{~mm}$ vs. $3.9 \pm 3.7 \mathrm{~mm}$, respectively). Compared with the control group, the recurrent LPD group showed significantly higher muscle-fibre angulation in the VMO $\left(35.4 \pm 8.0^{\circ}\right.$ vs. $\left.27.9 \pm 6.3^{\circ}\right)$. However, the craniocaudal extent of the VMO was significantly smaller in the LPD group than in the control group $(13.7 \pm 5.3 \mathrm{~mm}$ vs. $16.7 \pm 5.1 \mathrm{~mm})$. The average cross-sectional area ratio of the VMO was $5 \%$ in the recurrent LPD group and $7 \%$ in the control group (Table 2).
As shown in Fig. 4, normally, the line that passes through the deepest portion of the trochlear groove and was perpendicular to the posterior condylar reference line passed through near the midpoint of the widest plane of the patella (Fig. 4a). However, obvious patellar tilt and displacement were observed in the recurrent LPD group (Fig. 4b). Compared with the control group, the recurrent LPD group showed a significantly larger patella tilt angle $\left(25.9 \pm 10.7^{\circ}\right.$ vs. $\left.9.1 \pm 5.2^{\circ}\right)$; similarly, the bisect offset ratio of the LPD group was significantly higher than that of the control group $(0.97 \pm 0.33$ vs. $0.54 \pm 0.06)$.

\section{Discussion}

It is generally believed that patella maltracking usually manifests as the subluxation and outward displacement of the patella, which is mainly measured by the patella tilt angle and bisect offset ratio. The patella tilt angle is used to describe the degree of inclination of the patella, which is regarded as the most sensitive indicator to identify patella instability [26]. Lateral displacement of the patella was described by the bisect offset ratio, which was defined as the percentage of lateral width of the total 

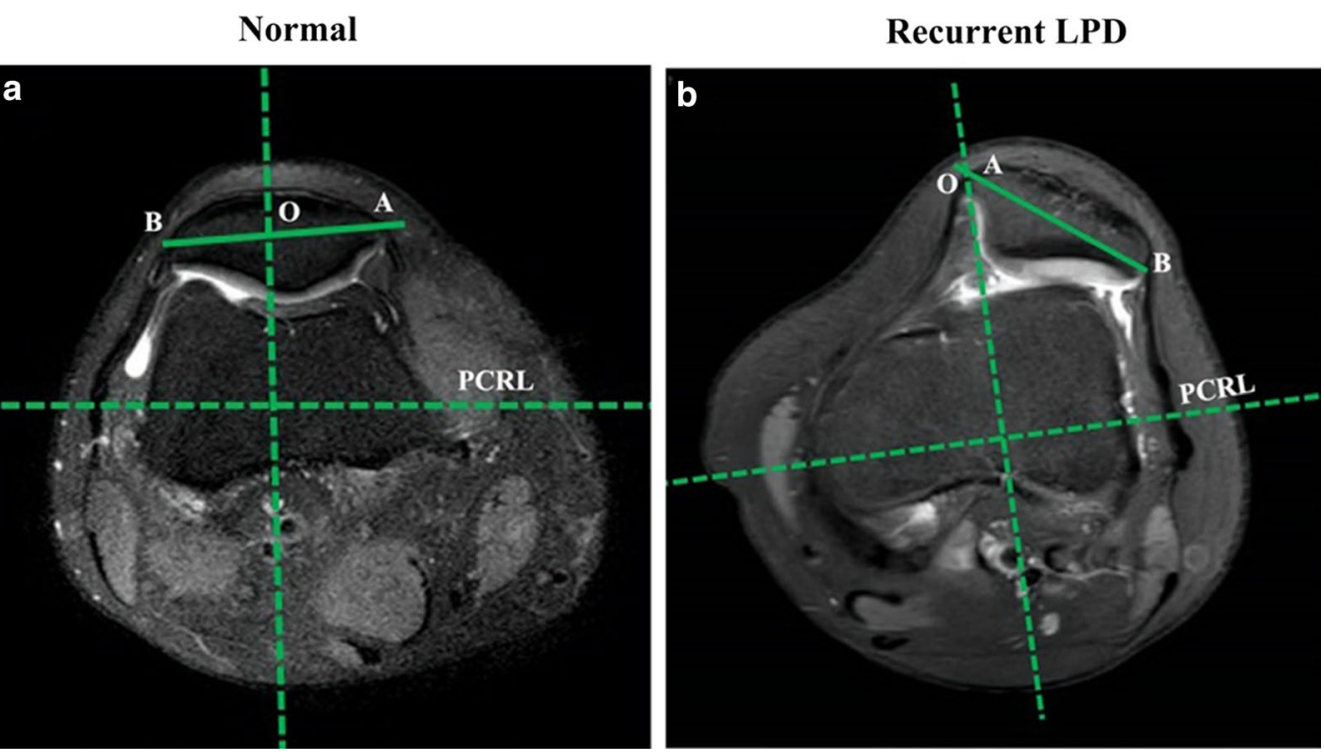

Fig. 4 The measurement of the patella tilt angle and bisect offset index in the control and recurrent LPD groups. The posterior condylar reference line (PCRL) was drawn tangent to the posterior femoral condyles. The tilt angle was measured as the angle between the PCRL (dashed line) and the line along the width of the maximal patella (solid line). A line was drawn through the deepest portion of the trochlear groove and perpendicular to the PCRL. The intersection of this line and the maximal patella width line was defined as point $\mathrm{O}$. In the transverse plane of the widest layer of the patella, the innermost point of the patella was defined as point $A$, and the outermost point was defined as point $B$. The ratio of OB/AB was defined as the bisect offset ratio

Table 1 The basic characteristics of the patients in the two groups

\begin{tabular}{|c|c|c|c|c|c|c|}
\hline \multirow[t]{2}{*}{ Group } & \multirow[t]{2}{*}{ Age, mean $\pm S D$ (range), y } & \multirow{2}{*}{$\begin{array}{l}\text { Sex (male/ } \\
\text { female) }\end{array}$} & \multirow{2}{*}{$\begin{array}{l}\text { BMI, mean } \pm S D \\
\left(\mathrm{~kg} / \mathrm{m}^{2}\right)\end{array}$} & \multicolumn{3}{|c|}{ Trochlear dysplasia (n) } \\
\hline & & & & Normal & Low-grade & High-grade \\
\hline Control & $24.0 \pm 6.5(19-38)$ & $25 / 45$ & $23.3 \pm 2.6$ & 57 & 18 & 0 \\
\hline Recurrent LPD & $23.1 \pm 9.9(12-45)$ & $18 / 36$ & $24.1 \pm 3.6$ & 0 & 16 & 59 \\
\hline$p$ & $>.05$ & $>.05$ & $>.05$ & $<.05$ & $>.05$ & $<.05$ \\
\hline
\end{tabular}

LPD lateral patellar dislocation

Table 2 Comparison of the study group and recurrent LPD group

\begin{tabular}{|c|c|c|c|c|c|c|c|}
\hline & \multicolumn{2}{|c|}{$\begin{array}{l}\text { Mean VMO elevation } \\
(\mathrm{mm})\end{array}$} & \multirow[t]{2}{*}{$\begin{array}{l}\text { Muscle-fibre } \\
\text { angulation }\left({ }^{\circ}\right)\end{array}$} & \multirow[t]{2}{*}{$\begin{array}{l}\text { Craniocaudal } \\
\text { extent }(\mathrm{mm})\end{array}$} & \multirow[t]{2}{*}{$\begin{array}{l}\text { Cross-sectional } \\
\text { area ratio }\end{array}$} & \multirow[t]{2}{*}{ Patella tilt angle $\left({ }^{\circ}\right)$} & \multirow[t]{2}{*}{ Bisect offset ratio } \\
\hline & Sagittal & Coronal & & & & & \\
\hline Control & $4.1 \pm 1.9$ & $3.9 \pm 3.7$ & $27.9 \pm 6.3$ & $16.7 \pm 5.1$ & $0.07 \pm 0.02$ & $9.1 \pm 5.2$ & $0.54 \pm 0.06$ \\
\hline Recurrent LPD & $10.4 \pm 2.3$ & $15.9 \pm 5.7$ & $35.4 \pm 8.0$ & $13.7 \pm 5.3$ & $0.05 \pm 0.02$ & $25.9 \pm 10.7$ & $0.97 \pm 0.33$ \\
\hline$P$ value & 0.00 & 0.00 & 0.00 & 0.005 & 0.000 & 0.00 & 0.00 \\
\hline
\end{tabular}

LPD Lateral patellar dislocation, VMO vastus medialis obliquus

patellar width [27]. Therefore, the patella tilt angle and bisect offset ratio were used to assess the patella position in patients with recurrent LPD in our study. As in the studies with conducted by Charles et al. [28] and Escala et al. [26], our study revealed a statistically significant difference in the patellar tilt angle between the control persons $\left(9^{\circ}\right)$ and recurrent LPD patients $\left(25^{\circ}\right)$. Moreover, another indicator of lateral tilt, the bisect offset ratio, showed a significant difference in two groups: the values were 0.54 and 0.97 in the control and LPD groups, 
respectively. The results mentioned above confirmed the existence of patellar inclination in recurrent LPD patients in our investigation. Femoral trochlear dysplasia has been recognized as a risk factor for patellar dislocation. The latest literature indicated that patellofemoral kinematics, stability, contact pressure, and contact area are significantly affected by trochlear dysplasia [29]. Trochleoplasty has also been shown to be effective in patients with severe trochlear dysplasia [30]. Our results also showed that high-grade trochlear dysplasia was more common in patients with recurrent LPD.

The VMO acts as a vital dynamic stabilizing device to limit the tendency of patellar dislocation. Anatomically, the VMO is basically perpendicular to the longitudinal axis of the patella sagittal position, thereby enhancing the stability of the patella [31]. The patella does not contact the trochlea at the beginning of knee flexion, and the tendency for patella dislocation is limited by the VMO and MPFL. An in vitro study revealed that when the VMO is weak, patellar displacement increases throughout $0-15^{\circ}$ of knee flexion [32]. Throughout $20^{\circ}-90^{\circ}$ of knee flexion, VMO relaxation can reduce the resistance of the patella lateral displacement by $30 \%$ [33]. Therefore, we consider that patellar tilt or lateral dislocation may be induced by abnormalities in the VMO. Until now, there has been no consensus on the role of the VMO in the stabilization of the patellofemoral joint, and some scholars have doubted that patellar instability is related to the VMO $[34,35]$. Nevertheless, more studies have shown that the VMO significantly affects patellofemoral instability. Pattyn et al. [15] suggested that VMO atrophy is present in patients with patellofemoral pain and is a contributing factor to patellofemoral pain syndrome. Moreover, the idea that the functional status of the VMO is closely related to recurrent LPD was verified in a diffusion tensor imaging study [36]. In a 2-year follow-up study, MPFL reconstructive surgery without VMO repair had no significant effect on re-dislocation in patients with primary patella dislocation compared with conservative treatment [37], which suggested that MPFL reconstruction combined with VMO repair may yield better postoperative outcomes. Zhang et al. [22] also suggested that more attention should be paid to the VMO, especially in those patients with complete femoral-side injuries. Hence, in preoperative evaluations, surgeons should carefully assess the injury conditions of the MPFL and VMO, and individualized treatment should be adopted for each patient.

In this study, we used elevation in the sagittal plane and coronal plane, cross-sectional area, muscle-fibre angulation and craniocaudal extent to comprehensively evaluate the morphological changes of the VMO in recurrent LPD patients. The VMO is originally attached intimately to the patella together with MPFL, and the presence of pathological elevation means that the $\mathrm{VMO}$ is no longer connected at the original attachment point to the patella or femur. The loss of the firm attachment to the distal origin, resulting in large sagittal and coronal elevations of the torn VMO muscle, may decrease the dynamic medial stabilizing force. This can be manifested by the elevation of the VMO in the coronal and sagittal planes, as observed in MRI scans, which may lead to a reduction in the limiting force of the VMO on the medial patellofemoral joint, thus increasing the extent of lateral inclination of the patella. This conclusion was confirmed in a cadaver study by Goh et al. [38], and comparisons showed that the VMO tension loss causes an increase in lateral displacement of the patella and increases the stress of the lateral patellar facet during knee flexion. Our results suggest that compared with individuals in the control group, patients with recurrent LPD have an VMO that is elevated by an average of about approximately $6 \mathrm{~mm}$ and $12 \mathrm{~mm}$ in the sagittal and coronal planes, respectively. These results indicated that the VMO is significantly elevated in recurrent LPD patients. However, the VMO was significantly elevated more in Zhang et al's study [22] than in our study. Elias et al. [20] found that approximately $55 \%$ of patients with acute LPD had significant effusion; thus, the average elevation of the VMO in the coronal plane was $2.2 \mathrm{~cm}$, which was also larger than that observed in our results, while the elevation in the sagittal plane was not noted. We speculate that this inconsistency may have been caused by massive effusions accumulating in the cavity after the first acute LPD, which leads an obvious degree of elevation of the VMO.

In addition, muscle-fibre angulation and craniocaudal extent may also have an influence on the tension of the VMO, and a cadaver study has shown that the absence of VMO tension leads to a lateral shift of the patella [38]. Our results showed that the muscle-fibre angulation and craniocaudal extent of the VMO significantly differed between the two groups, the muscle-fibre angle was larger by an average of $12^{\circ}$ and craniocaudal extent was smaller by $3 \mathrm{~mm}$ in the LPD group than in the control group. In our investigation, the average craniocaudal extent was $13.7 \mathrm{~mm}$ in patients with recurrent LPD, which was consistent with the average of $14 \mathrm{~mm}$ reported in Balcarek et al's study [23]. Previous cadaver studies have indicated that muscle-fibre angulation of the $\mathrm{VMO}$ in normal people ranges from $42^{\circ}$ to $52^{\circ}$ [31]; however, the average muscle-fibre angulation in the control group and recurrent LPD patients were $27^{\circ}$ and $35^{\circ}$ in our study, respectively. Balcarek et al. [23] found that the muscle-fibre angulation in the control group was $44^{\circ}-48^{\circ}$, the prevalence of recurrent LPD females subjects in our study was $33.3 \%(18 / 54)$ while in their study was $50 \%$ and there was also a difference in the 
BMI of the population, so we think this discrepancy might be related to the study population. Moreover, in the LPD group in their study, this angle was smaller than that in the control group (no significant statistical difference), but we obtained the opposite result. In fact, it seems plausible that the VMO will tear or injure in femoral attachment points after LPD, causing the muscle to move away from adductor tubercle. Therefore, the muscle fibres of the VMO will shift forward and upward relative to the longitudinal axis of femur in the sagittal plane, which will inevitably lead to an increase rather than a decrease in muscle-fibre angulation (Fig. 2b, e).To date, no similar studies have been conducted, so the normal range of muscle-fibre angulation of the VMO remains to be studied further.

It has been proven that the cross-sectional area can be used to assess the force-producing ability of muscle and can be measured on MR images $[39,40]$. To simulate the three-dimensional structure of the muscle, three adjacent layers of the upper edge of the patella were selected to calculate the cross-sectional area of the VMO (Fig. 3). Furthermore, we calculated the ratio between the crosssectional area of the VMO and the whole thigh to obtain the volume proportion of the VMO and minimize individual differences. As a result, the ratio of the VMO cross-sectional area of the corresponding thigh area in the recurrent LPD group was significantly lower than that in the control group. Both Balcarek et al. [23] and Liu et al. [36] found that the cross-sectional area of the VMO showed a decreasing trend in patients with recurrent PLD, but the differences between these patients and healthy volunteers were not significant; this lack of statistical significance may be related to the small sample size, as there were only 30 cases of LPD group in both studies. Another reason may be that we used the ratio to personalize the cross-sectional areas, which are more likely to show significant differences.

At present, there are no widely accepted quantitative indicators to evaluate VMO injuries on MRI. Zhang et al. [22] found that probability of injury of the VMO was $47.7 \%$ in patients with first patellar dislocation, indicating that nearly half of the patients with acute first LPD had a VMO injury. In this study, the recurrent LPD patients we included did not have a history of acute trauma in the short term, and the proportion of VMO injuries in the recurrent LPD group was only $24 \%$ (18/75). Therefore, in patients with recurrent LPD, it is difficult to determine whether the VMO is injured on the basis of only the presence of haemorrhage and oedema in MRI images. We attempted to use the above five quantitative indicators to evaluate VMO abnormalities, which can be caused by congenital factors or incomplete healing due to the failure to seek treatment after the initial dislocation.
Nonetheless, the results above should be interpreted with consideration of the limitations of this investigation. First, as this study was retrospective, the patients with recurrent LPD had obvious anatomical abnormalities, so the assessors could not be blinded to the diagnoses of the patients during the assessments. Second, our study indicated only that the VMO is abnormal in recurrent LPD patients, while it did not reveal whether the abnormality is a contributing factor or secondary to recurrent LPD. Third, the static morphological characteristics of only the VMO were studied, and we did not assess the vastus lateralis muscle; the balance between the two muscles may be important for evaluating the role of the VMO in maintaining patella stability. Moreover, recurrent LPD is often the result of multiple factors. To determine the role of the VMO in recurrent LPD patients, other factors (e.g., trochlear dysplasia, patella alta, and an increased tibial tuberosity-trochlear groove distance) for patellar dislocation were not analysed, so a more detailed and comprehensive assessment remains to be completed.

\section{Conclusions}

In conclusion, the results showed that abnormalities of the VMO were clearly present in recurrent LPD patients compared with normal people. MRI was used to comprehensively evaluate the morphological parameters of the VMO in patients with recurrent LPD, and the presence of VMO elevation in the coronal and sagittal planes, a low cross-sectional area ratio, a high muscle-fibre angulation and a small craniocaudal extent may be related to patellar inclination. Based on the results of previous biomechanical and clinical studies, we summarized the methods for evaluating the VMO by MRI, which is helpful for clinicians to identify VMO atrophy by objective morphological parameters. Moreover, by using the individualized index (cross-sectional area ratio of the VMO and bisect offset ratio), we can better to reduce the measuring errors and evaluated the abnormalities of the VMO and patella deviation.

\section{Abbreviations \\ FOV: Field of view; ICC: Intraclass correlation coefficient; LPD: Lateral patella dislocation; MRI: Magnetic resonance imaging; MPFL: Medial patellofemoral ligament; PACS: Picture archiving and communications system; PDW-SPAIR: Proton density weighted spectral attenuated inversion recovery; PDW-SPIR: Pproton density weighted spectral inversion recovery; TR: Time msec; TE: Time msec; TSE: Turbo spin-echo; VMO: Vastus medialis obliquus; VL: Vastus lateralis.}

\section{Acknowledgements}

We sincerely appreciate all the patients who participated in this study.

\section{Author contributions}

This study was developed by CLB and NQB. The first draft of the manuscript was written by SL. All authors (SL, YX, HHY, CB, CLB, NQB) contributed to interpretation of the results and critical revision of the manuscript. All authors have approved the final manuscript before submission. 


\section{Funding}

This research was financially supported by the National Natural Science Foundation of China (NO. 81803275) in estimate the feasibility of the study. The funding played no role in the design of the study and collection, analysis, and interpretation of data and in writing the manuscript.

\section{Availability of data and materials}

The data for this study are provided in the Tables of the main paper. Due to patient privacy protection, other additional materials and data are not publicly available but are available from the corresponding author on reasonable request.

\section{Ethics approval and consent to participate}

The Ethics Committee of Wuhan University Zhongnan Hospital approved this retrospective study and waived the requirement for written informed consent due to its retrospective nature.

\section{Consent for publication}

Not applicable.

\section{Competing interests}

The authors declare that they have no competing interests.

Received: 29 July 2020 Accepted: 22 December 2020

Published online: 06 January 2021

\section{References}

1. Colvin AC, West RV. Patellar instability. J Bone Joint Surg Am. 2008;90(12):2751-62.

2. Gravesen KS, Kallemose T, Blønd L, Troelsen A, Barfod KW. High incidence of acute and recurrent patellar dislocations: a retrospective nationwide epidemiological study involving 24.154 primary dislocations. Knee Surg Sports Traumatol Arthrosc. 2018;26(4):1204-9.

3. Tan SHS, Ibrahim MM, Lee ZJ, Chee YKM, Hui JH. Patellar tracking should be taken into account when measuring radiographic parameters for recurrent patellar instability. Knee Surg Sports Traumatol Arthrosc. 2018;26(12):3593-600

4. Hawkins RJ, Bell RH, Anisette G. Acute patellar dislocations. The natural history. Am J Sports Med. 1986;14(2):117-20.

5. Stefancin JJ, Parker RD. First-time traumatic patellar dislocation: a systematic review. Clin Orthop Relat Res. 2007;455:93-101.

6. Petri M, von Falck C, Broese M, Liodakis E, Balcarek P, Niemeyer P, et al. Influence of rupture patterns of the medial patellofemoral ligament (MPFL) on the outcome after operative treatment of traumatic patellar dislocation. Knee Surg Sports Traumatol Arthrosc. 2013;21(3):683-9.

7. Philippot R, Boyer B, Testa R, Farizon F, Moyen B. The role of the medial ligamentous structures on patellar tracking during knee flexion. Knee Surg Sports Traumatol Arthrosc. 2012;20(2):331-6.

8. Panagiotopoulos E, Strzelczyk P, Herrmann M, Scuderi G. Cadaveric study on static medial patellar stabilizers: the dynamizing role of the vastus medialis obliquus on medial patellofemoral ligament. Knee Surg Sports Traumatol Arthrosc. 2006;14(1):7-12.

9. Brunet ME, Brinker MR, Cook SD, Christakis P, Fong B, Patron L, et al. Patellar tracking during simulated quadriceps contraction. Clin Orthop Relat Res. 2003:414:266-75

10. Sarkar A, Razdan S, Yadav J, Bansal N, Kuhar S, Pahuja P. Effect of isometric quadricep activation on " $Q$ " angle in young females. Indian J Physiol Pharmacol. 2009;53(3):275-8.

11. Hinckel BB, Gobbi RG, Kihara Filho EN, Demange MK, Pécora JR, Camanho GL. Patellar tendon-trochlear groove angle measurement: a new method for patellofemoral rotational analyses. Orthop J Sports Med. 2015;3(9):2325967115601031.

12. Hinckel BB, Gobbi RG, Kihara Filho EN, Demange MK, Pécora JR, Rodrigues $M B$, et al. Why are bone and soft tissue measurements of the TT-TG distance on MRI different in patients with patellar instability? Knee Surg Sports Traumatol Arthrosc. 2017;25(10):3053-60.

13. Pal S, Besier TF, Draper CE, Fredericson M, Gold GE, Beaupre GS, et al. Patellar tilt correlates with vastus lateralis: vastus medialis activation ratio in maltracking patellofemoral pain patients. J Orthop Res. 2012;30(6):927-33.

14. Pal S, Draper CE, Fredericson M, Gold GE, Delp SL, Beaupre GS, et al. Patellar maltracking correlates with vastus medialis activation delay in patellofemoral pain patients. Am J Sports Med. 2011;39(3):590-8.

15. Pattyn E, Verdonk P, Steyaert A, Vanden Bossche L, Van den Broecke W, Thijs Y, et al. Vastus medialis obliquus atrophy: does it exist in patellofemoral pain syndrome? Am J Sports Med. 2011;39(7):1450-5.

16. Seeley M, Bowman KF, Walsh C, Sabb BJ, Vanderhave KL. Magnetic resonance imaging of acute patellar dislocation in children: patterns of injury and risk factors for recurrence. J Pediatr Orthop. 2012;32(2):145-55.

17. Samim M, Smitaman E, Lawrence D, Moukaddam H. MRI of anterior knee pain. Skelet Radiol. 2014;43(7):875-93.

18. Dejour D, Saggin P. The sulcus deepening trochleoplasty-the Lyon's procedure. Int Orthop. 2010;34(2):311-6.

19. Lippacher S, Dejour D, Elsharkawi M, Dornacher D, Ring C, Dreyhaupt J, et al. Observer agreement on the Dejour trochlear dysplasia classification: a comparison of true lateral radiographs and axial magnetic resonance images. Am J Sports Med. 2012;40(4):837-43.

20. Elias DA, White LM, Fithian DC. Acute lateral patellar dislocation at MR imaging: injury patterns of medial patellar soft-tissue restraints and osteochondral injuries of the inferomedial patella. Radiology. 2002;225(3):736-43.

21. Shu L, Ni Q, Yang X, Chen B, Wang H, Chen L. Comparative study of the tibial tubercle-trochlear groove distance measured in two ways and tibial tubercle-posterior cruciate ligament distance in patients with patellofemoral instability. J Orthop Surg Res. 2020;15(1):209.

22. Zhang G-Y, Zheng L, Shi H, Liu W, Zhang L, Qu S-H, et al. Correlation analysis between injury patterns of medial patellofemoral ligament and vastus medialis obliquus after acute first-time lateral patellar dislocation. Knee Surg Sports Traumatol Arthrosc. 2018;26(3):719-26.

23. Balcarek $P$, Oberthür $S$, Frosch $S$, Schüttrumpf JP, Stürmer KM. Vastus medialis obliquus muscle morphology in primary and recurrent lateral patellar instability. Biomed Res Int. 2014;2014:326586.

24. Powers CM, Shellock FG, Pfaff M. Quantification of patellar tracking using kinematic MRI. J Magn Reson Imaging. 1998;8(3):724-32.

25. Callaghan MJ, Guney H, Reeves ND, Bailey D, Doslikova K, Maganaris CN, et al. A knee brace alters patella position in patellofemoral osteoarthritis: a study using weight bearing magnetic resonance imaging. Osteoarthr Cartil. 2016;24(12):2055-60.

26. Escala JS, Mellado JM, Olona M, Giné J, Saurí A, Neyret P. Objective patellar instability: MR-based quantitative assessment of potentially associated anatomical features. Knee Surg Sports Traumatol Arthrosc 2006;14(3):264-72.

27. Draper CE, Besier TF, Fredericson M, Santos JM, Beaupre GS, Delp SL, et al. Differences in patellofemoral kinematics between weight-bearing and non-weight-bearing conditions in patients with patellofemoral pain. J Orthop Res. 2011;29(3):312-7.

28. Charles MD, Haloman S, Chen L, Ward SR, Fithian D, Afra R. Magnetic resonance imaging-based topographical differences between control and recurrent patellofemoral instability patients. Am J Sports Med. 2013:41(2):374-84.

29. Van Haver A, De Roo K, De Beule M, Labey L, De Baets P, Dejour D, et al. The effect of trochlear dysplasia on patellofemoral biomechanics: a cadaveric study with simulated trochlear deformities. Am J Sports Med. 2015:43(6):1354-61.

30. Carstensen SE, Feeley SM, Burrus MT, Deasey M, Rush J, Diduch DR. Sulcus deepening trochleoplasty and medial patellofemoral ligament reconstruction for patellofemoral instability: a 2-year study. Arthroscopy. 2020;36(8):2237-45.

31. Farahmand F, Senavongse W, Amis AA. Quantitative study of the quadriceps muscles and trochlear groove geometry related to instability of the patellofemoral joint. J Orthop Res. 1998;16(1):136-43.

32. Sakai N, Luo ZP, Rand JA, An KN. The influence of weakness in the vastus medialis oblique muscle on the patellofemoral joint: an in vitro biomechanical study. Clin Biomech (Bristol, Avon). 2000;15(5):335-9.

33. Senavongse $W$, Amis AA. The effects of articular, retinacular, or muscular deficiencies on patellofemoral joint stability: a biomechanical study in vitro. J Bone Joint Surg Br. 2005;87(4):577-82. 
34. Powers CM, Landel R, Perry J. Timing and intensity of vastus muscle activity during functional activities in subjects with and without patellofemoral pain. Phys Ther. 1996;76(9):946-55.

35. Pattyn E, Verdonk P, Steyaert A, Van Tiggelen D, Witvrouw E. Muscle functional MRI to evaluate quadriceps dysfunction in patellofemoral pain. Med Sci Sports Exerc. 2013;45(6):1023-9.

36. Liu L-S, Zheng Z-Z, Yuan H-S. Significance of diffusion tensor imaging of vastus medialis oblique in recurrent patellar dislocation. Chin Med J. 2017:130(6):642-6.

37. Christiansen SE, Jakobsen BW, Lund B, Lind M. Isolated repair of the medial patellofemoral ligament in primary dislocation of the patella: a prospective randomized study. Arthroscopy. 2008;24(8):881-7.

38. Goh JC, Lee PY, Bose K. A cadaver study of the function of the oblique part of vastus medialis. J Bone Joint Surg Br. 1995;77(2):225-31.
39. Bamman MM, Newcomer BR, Larson-Meyer DE, Weinsier RL, Hunter GR. Evaluation of the strength-size relationship in vivo using various muscle size indices. Med Sci Sports Exerc. 2000;32(7):1307-13.

40. Frontera WR, Hughes VA, Fielding RA, Fiatarone MA, Evans WJ, Roubenoff R. Aging of skeletal muscle: a 12-yr longitudinal study. J Appl Physiol. 2000;88(4):1321-6.

\section{Publisher's Note}

Springer Nature remains neutral with regard to jurisdictional claims in published maps and institutional affiliations.
Ready to submit your research? Choose BMC and benefit from:

- fast, convenient online submission

- thorough peer review by experienced researchers in your field

- rapid publication on acceptance

- support for research data, including large and complex data types

- gold Open Access which fosters wider collaboration and increased citations

- maximum visibility for your research: over $100 \mathrm{M}$ website views per year

At BMC, research is always in progress.

Learn more biomedcentral.com/submissions 